\title{
Repérage des cas d'insuffisance cardiaque congestive à partir de données administratives : étude de validation utilisant des dossiers de patients en soins primaires
}

\author{
S. E. Schultz, M.A., M. Sc. (1); D. M. Rothwell, M. Sc. (2); Z. Chen, M.D. (1); K. Tu, M.D. (1, 3, 4)
}

Cet article a fait l'objet d'une évaluation par les pairs.

\section{Résumé}

Introduction : En vue de déterminer si l'utilisation conjuguée des données administratives hospitalières et des factures présentées par les médecins au titre des soins ambulatoires pouvait permettre de repérer avec exactitude les patients souffrant d'insuffisance cardiaque congestive (ICC), nous avons mis à l'essai neuf algorithmes pour repérer à partir de données administratives les personnes souffrant d'ICC.

Méthodologie : La cohorte de validation par rapport à laquelle les essais ont été effectués combinait des données provenant d'un échantillon aléatoire de patients adultes inscrits dans la base de données EMRALD de dossiers médicaux électroniques des médecins de premier recours en Ontario, au Canada, et des données recueillies en 2004-2005 à partir d'un échantillon aléatoire de patients en soins primaires pour une étude sur l'hypertension. On a évalué la sensibilité, la spécificité, la valeur prédictive positive, l'aire sous la courbe ROC et la combinaison des rapports de vraisemblance positif et négatif des algorithmes.

Résultats : Nous avons constaté qu'un dossier d'hospitalisation ou de facturation de médecin suivi d'un second dossier provenant de l'une ou l'autre de ces sources dans la même année produisait les meilleurs résultats, avec une sensibilité de $84,8 \%$ et une spécificité de 97,0\%.

Conclusion : La prévalence de l'ICC dans la population peut être mesurée avec exactitude à partir de données administratives issues de l'hospitalisation et des soins ambulatoires.

Mots-clés : insuffisance cardiaque congestive, études de validation, méthodes épidémiologiques, prévalence dans la population

\section{Introduction}

Les dossiers de congé des hôpitaux ${ }^{1,2}$ servent depuis longtemps à repérer les patients souffrant d'insuffisance cardiaque congestive (ICC) qui se présentent à l'hôpital, ou qui sont hospitalisés pour d'autres problèmes de santé mais dont le dossier mentionne l'ICC à titre d'affection comorbide. Dans leur examen systématique récent des études de validation des algorithmes utilisés pour repérer les patients atteints d'ICC à partir de données administratives, Saczynski et collab. ${ }^{3}$ ont observé que cela était vrai pour 25 des 35 études répertoriées.

Il a été démontré que l'utilisation des dossiers de congé des hôpitaux par rapport à celle des dossiers d'hospitalisation pour repérer les patients atteints d'ICC donnait des résultats d'une grande exactitude ${ }^{4,5}$. Toutefois, compte tenu de l'amélioration des traitements et de la raréfaction des ressources hospitalières, une prise en charge efficace dans la collectivité des patients souffrant d'insuffisance cardiaque est de plus en plus fréquente. Par conséquent, ces patients n'apparaitront probablement pas dans les données sur les congés des hôpitaux, ou alors uniquement lorsque leur maladie aura atteint un stade avancé. En n'utilisant que les données sur les hospitalisations, on sous-estime donc probablement l'incidence et la prévalence de l'ICC.

On a mis au point et validé des algorithmes faisant appel à la fois aux données de facturation des médecins et aux dossiers de congé des hôpitaux en vue de repérer les patients présentant une affection chronique qui n'exige pas d'hospitalisation, comme l'hypertension, le diabète, la cardiopathie ischémique et l'asthme ${ }^{6,9}$. Toutefois, parmi les 35 études répertoriées dans l'examen systématique effectué par Saczynski et collab. ${ }^{3}$, neuf seulement utilisaient des données provenant à la fois des dossiers de congé des hôpitaux et des demandes de paiement des soins ambulatoires, et deux seulement utilisaient également des données sur une population, même si cette population était limitée aux patients affiliés à un important organisme de gestion de soins ${ }^{10,11}$.

L’objet de notre étude était de déterminer l'algorithme de données administratives le plus approprié pour repérer les patients

Rattachement des auteurs :

1. Institut de recherche en services de santé, Toronto (Ontario), Canada

2. Institut de recherche de l'Hôpital d'Ottawa, Ottawa (Ontario), Canada

3. Département de médecine familiale et communautaire, Université de Toronto, Toronto (Ontario), Canada

4. Équipe de santé familiale de l'Hôpital Toronto Western, Réseau universitaire de santé, Toronto (Ontario), Canada

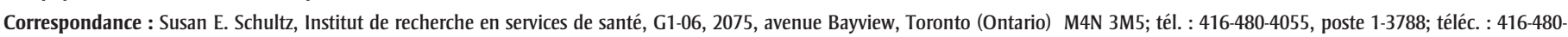
6048; courriel : sue.schultz@ices.on.ca 
atteints d'ICC en Ontario (Canada). Nous avons utilisé l'information disponible dans les dossiers médicaux électroniques (DME) des patients vus par des médecins de premier recours en consultation externe et dans les dossiers des médecins de premier recours payés à l'acte afin d'évaluer la validité et la fiabilité de différentes combinaisons de données de facturation des médecins et des données des hôpitaux sur les congés.

\section{Méthodologie}

\section{Sources des données}

\section{Cohorte de validation}

La cohorte de validation utilisée dans cette étude comprenait des données provenant de deux sources. La première était celle des données recueillies par l'Équipe canadienne de recherche sur les résultats des interventions en santé cardiovasculaire (Canadian Cardiovascular Outcomes Research Team, ou CCORT), par l'intermédiaire de 17 médecins ayant utilisé le logiciel de DME de Solutions cliniques ${ }^{\circledR}$ et ayant transmis les dossiers de leurs patients à la base de données EMRALD (Electronic Medical Record Administrative data Linked Database). Les médecins participant à cette étude devaient avoir utilisé les DME depuis au minimum deux ans afin que tous leurs patients possèdent un DME. Les données des DME ont été extraites entre juin et décembre 2007, puis ont été anonymisées, chiffrées, et transférées par voie électronique sécurisée à l'Institut de recherche en services de santé (IRSS). L'IRSS est une entité visée par la Loi sur la protection des renseignements personnels sur la santé de l'Ontario, ce qui signifie que cette organisation peut, sans obtenir de consentement, recevoir et utiliser des renseignements sur la santé à des fins d'analyse et de compilation de données statistiques sur le système de soins de santé de l'Ontario. La collecte des données se fait à partir d'un large éventail de sources, dont le ministère de la Santé et des Soins de longue durée, les hôpitaux et les médecins, dans la mesure où l'IRSS a mis en place des politiques, des pratiques et des procédures qui ont été vérifiées et approuvées par le Commissaire à l'information et à la protection de la vie privée de l'Ontario. Les données utilisées dans la présente étude ont été traitées conformément à la méthode de fonctionnement uniformisée de l'organisme visant à assurer le respect de la vie privée et de la confidentialité des données des patients.

La population totale admissible de patients ayant un DME était constituée de 19376 patients adultes actifs de 20 ans ou plus. Le terme " actif » signifie ici inscrit auprès d'un des médecins participants, ayant consulté au moins deux fois au cours des trois dernières années et possédant une carte valide de l'Assurancesanté de l'Ontario. Trois analystes spécialistes de l'extraction de données ont extrait les données à partir d'un échantillon aléatoire de $5 \%$ de ces patients $(n=969)$. La fidélité inter-observateurs touchant la concordance globale de la présence ou de l'absence d'ICC était très satisfaisante (indice kappa $[\kappa]>0,80$ ).

La seconde source de données était un échantillon aléatoire de dossiers de patients de 76 cabinets de médecins de famille rémunérés à l'acte dont les données avaient été extraites entre décembre 2004 et août 2005 pour valider un algorithme faisant appel aux données administratives afin de repérer les cas d'hypertension ${ }^{6}$. Dans cette étude, l'extraction des données des dossiers a été effectuée à partir d'un échantillon aléatoire de tous les patients admissibles $(n=2$ 472). Étaient admissibles les patients réguliers du cabinet médical qui étaient venus en consultation au moins deux fois au cours des trois années précédentes, qui étaient âgés d'au moins 38 ans et qui possédaient un numéro de carte santé de l'Ontario. Deux spécialistes ont extrait les données des dossiers, ici encore avec une très bonne fidélité interobservateurs à l'égard de la concordance globale de la présence ou de l'absence d'ICC $(\kappa>0,80)$.

Nous avons repéré par la même méthode les patients atteints d'ICC à partir des deux sources de données : des spécialistes de l'extraction des données des dossiers ont examiné toutes les entrées figurant dans le DME ou, durant les trois années précédentes, dans le dossier médical des patients. Pour chaque entrée, on a indiqué s'il y avait présence d'ICC, possibilité
d'ICC, antécédents familiaux d'ICC ou absence d'ICC. Une entrée pour laquelle on notait la présence d'ICC signifiait que le médecin avait explicitement indiqué dans le dossier que le patient était atteint d'ICC ou d'insuffisance ventriculaire, de décompensation cardiaque, d'insuffisance cardiaque (droite ou gauche), de dysfonctionnement de la pompe cardiaque, d'insuffisance ventriculaire ou d'œdème pulmonaire, qui sont autant de synonymes de l'ICC. Pour les résultats des tests diagnostiques compatibles avec une conclusion d'ICC, on a indiqué qu'il y avait possibilité d'ICC. S'il n'y avait aucune mention de l'ICC ou de l'un de ses synonymes dans le dossier, on a considéré que le patient n'était pas atteint d'ICC. Lors de la compilation des données, le patient n'était considéré comme atteint d'ICC que si une ou plusieurs entrées confirmaient la présence de cette affection.

Pour assurer la cohérence entre les deux sources de données, seuls les individus appartenant à la cohorte de DME âgés d'au moins 38 ans ont été inclus. Nous ne nous attendions pas à ce que cela introduise un biais quelconque, car l'échantillon de DME était un simple échantillon aléatoire de chaque cabinet médical. Pour permettre l'analyse de ces données, on a chiffré le numéro de la carte santé de chaque patient afin d'obtenir un numéro d'identification unique pouvant être apparié avec les fonds de données administratives de l'Ontario hébergées à l'IRSS.

\section{Sources de données administratives}

Les sources des données administratives utilisées pour repérer les cas d'ICC dans la population étaient la Base de données sur les congés des patients (BDCP) et la Base de données sur les chirurgies d'un jour (SDS), hébergées par l'Institut canadien d'information sur la santé (ICIS), ainsi que la base de données de l'Assurance santé de l'Ontario des factures des médecins rémunérés à l'acte ou des factures pro forma. Dans les bases de données BDCP et SDS, les diagnostics sont classifiés à l'aide des codes de la Classification internationale des maladies, $9^{e}$ révision (CIM-9) s'ils sont antérieurs à 2002 et à l'aide des codes de la $10^{e}$ révision s'ils sont postérieurs à 2002; la base de données de l'Assurancesanté de l'Ontario utilise une 
version modifiée de la CIM-8. La base de données sur la facturation des médecins de l'Assurancesanté de l'Ontario consigne plus de $95 \%$ des consultations de médecins de premier recours exerçant en cabinet par les résidants de l'Ontario. Le code de diagnostic correspondant à l'ICC dans la base de données de l'sssurancesanté de l'Ontario sur la facturation des médecins ou dans les bases de données de l'ICIS sur l'hospitalisation (BDCP ou SDS) était le code du diagnostic principal; à défaut de ce code, une affection comorbide était considérée comme une indication positive de la présence d'ICC.

\section{Codes de diagnostic utilisés pour définir I'ICC}

Les codes de diagnostic utilisés pour définir l'ICC varient considérablement. Dans la CIM-9, l'ICC est le plus souvent définie par le code 428 . Lee et collab. ${ }^{4}$ ont validé le code 428 de la CIM-9 par rapport à deux ensembles de critères cliniques en utilisant les données des dossiers des hôpitaux sur les patients et ont conclu qu'il était hautement prédictif. Vermeulen et collab. ${ }^{13}$ ont eux aussi comparé l'utilité des codes 428 de la CIM- 9 et I50 de la CIM10 et ont conclu qu'ils étaient comparables. Ces deux résultats répondent à la question de savoir si les codes 428 de la CIM-9 et I50 de la CIM-10 permettent de repérer correctement les cas d'insuffisance cardiaque. Toutefois, ils ne permettent pas de déterminer si ces codes sont suffisants pour repérer tous les cas d'ICC. Dans des études effectuées ailleurs ${ }^{5,14,15}$, on a utilisé une palette plus large de codes de diagnostic pour repérer les cas d'ICC à partir des données administratives. Nous avons décidé de comparer deux définitions de l'ICC : une définition étroite utilisant uniquement le code 428 de la CIM-9 et les codes I500, I501 et I509 de la CIM-10, et une définition plus large incluant également les codes correspondant à la myocardiopathie (codes 425 de la CIM-9 et I42 de la CIM-10) et à l'œdème pulmonaire (codes 514 et 518.4 de la CIM-9 et J81 de la CIM-10).

\section{Algorithmes évalués}

Nous avons évalué neuf algorithmes, qui variaient du point de vue des sources de données utilisées et de la durée du suivi. La performance des différents algorithmes de données administratives a été évaluée par rapport au statut à l'égard de l'ICC obtenu par extraction manuelle des données du dossier ou du DME des patients. Ces algorithmes peuvent être divisés en trois grands groupes. Le premier, constitué des algorithmes 1 à 3 , ne nécessite qu'un dossier pour le diagnostic d'ICC, mais permet une évaluation comparative de l'utilisation de différentes sources de données. Les algorithmes 4 à 6 nécessitent soit un dossier d'hospitalisation, soit un dossier de soins ambulatoires et un dossier additionnel de l'une ou l'autre source à l'intérieur d'une période donnée pouvant être de un, de deux ou de trois ans. Les algorithmes 7 à 9, qui constituent le troisième groupe, sont similaires aux algorithmes 4 à 6 , mais utilisent uniquement des données sur les soins ambulatoires et nécessitent deux dossiers de cette catégorie à l'intérieur d'une période de suivi de un, de deux ou de trois ans (tableau 1).

Nous avons évalué chaque algorithme en ce qui concerne sa sensibilité, sa spécificité et sa valeur prédictive positive (VPP) et avons calculé les intervalles de confiance (IC) à $95 \%$ par la méthode d'approximation binomiale. Toutes les analyses ont été effectuées à l'aide du logiciel SAS, version 9.2 (SAS Institute, Cary, Caroline du Nord, États-Unis).
Nous avons également estimé deux mesures sommaires, la courbe caractéristique de la performance du test (courbe ROC) et les rapports de vraisemblance positif et négatif (RV+ et $R V-$ ) afin de préciser l'évaluation du diagnostic. La courbe ROC, d'abord élaborée pour évaluer la détection des signaux par les opérateurs de radars, est une représentation graphique de la relation entre le taux de vrais positifs (sensibilité) et celui de faux positifs (1 - spécificité). Plus l'aire sous la courbe (ASC) se rapproche de la valeur de 1,00, meilleur est le test.

La représentation graphique du rapport de vraisemblance indique la relation entre le $\mathrm{RV}+$, qui est le rapport du taux de vrais positifs (sensibilité) sur celui de faux positifs (1 - spécificité), et le RV-, qui est le rapport du taux de faux négatifs (1 sensibilité) sur celui de vrais négatifs (spécificité) ${ }^{16}$. Le RV+ mesure la capacité d'un test d'inclure les personnes atteintes de la maladie visée, tandis que le RVmesure sa capacité d'exclure les personnes qui n'en sont pas atteintes. Si l'on utilise les seuils proposés pour la première fois par Jaeschke et collab. ${ }^{17}$, les tests dont le $\mathrm{RV}+$ est supérieur à 10 et dont le RV- est inférieur à 0,1 sont considérés comme étant très utiles, tandis que ceux dont le $\mathrm{RV}+$ est compris entre 5 et 10 et dont le $\mathrm{RV}$ - est compris entre 0,1 et 0,2 sont considérés comme modérément utiles, et ceux dont le RV+ est compris entre 2 et 5 et le RV- est compris entre 0,2 et 0,5 ne

\section{TABLEAU 1}

Algorithmes fondés sur des données administratives évalués par rapport au statut à l'égard de I'ICC obtenu par extraction manuelle des données du dossier ou du DME des patients

\begin{tabular}{ll} 
Algorithme & Description \\
\hline 1 & 1 dossier d'hospitalisation \\
2 & 1 dossier de soins ambulatoires \\
3 & 1 dossier d'hospitalisation ou de soins ambulatoires \\
4 & 1 dossier d'hospitalisation seulement ou 1 dossier de soins ambulatoires suivi d'un \\
& autre dossier dans l'année suivante \\
5 & 1 dossier d'hospitalisation seulement 0 d 1 dossier de soins ambulatoires suivi d'un \\
6 & $\begin{array}{l}1 \text { dossier d'hospitalisation seulement ou } 1 \text { dossier de soins ambulatoires suivi d'un } \\
\text { autre dossier dans les } 3 \text { années suivantes }\end{array}$ \\
7 & 2 dossiers de soins ambulatoires en 1 an \\
9 & 2 dossiers de soins ambulatoires en 2 ans \\
\hline
\end{tabular}

Abréviations : DME, dossier médical électronique; ICC, insuffisance cardiaque congestive. 
sont que peu utiles. Les tests dont le RV+ est inférieur à 2 et dont le RV- est supérieur à 0,5 ne sont d'aucune utilité.

\section{Résultats}

La cohorte de validation combinée comptait 2338 patients, dont 99 atteints d'ICC d'après leur dossier médical ou leur DME et 2239 non atteints (prévalence : 4,2\%).

La distribution selon l'âge et le sexe des patients dans la cohorte de validation était similaire à celle de la population de l'Ontario en $2006^{18}$ âgée d'au moins 38 ans, avec une légère surreprésentation des femmes (56 \% dans la cohorte comparativement à $52 \%$ dans la population générale) et des personnes âgées de 65 ans et plus (32 \% dans la cohorte comparativement à $26 \%$ dans la population de l'Ontario âgée de 38 ans et plus). Cette situation était attendue, car notre cohorte de validation était composée de personnes qui consultent régulièrement un médecin de famille, et l'on sait que tant les femmes que les personnes âgées sont plus susceptibles de consulter un médecin ${ }^{19}$. L'âge moyen de notre cohorte d'adultes actifs était de 57,9 ans, soit un peu plus que l'âge moyen (56,1 ans) des Ontariens adultes âgés de 38 ans et plus.

Si l'on considère les trois groupes d'algorithmes de façon générale (voir le tableau 2), deux caractéristiques se détachent nettement. La première est que la durée du suivi n’a que peu d'effet. Les résultats pour les algorithmes 4,5 et 6 sont pratiquement identiques et, de même, il n'y a qu'une légère différence entre la performance des algorithmes 7, 8 et 9. Ce qui semble avoir plus d'effets est le choix des sources de données. Par exemple, la sensibilité des algorithmes 4,5 ou 6 , qui utilisent à la fois les données sur l'hospitalisation et celles sur les soins ambulatoires, est supérieure d'au moins 10 points de pourcentage à celle des algorithmes 7, 8 ou 9, qui ne font appel qu'aux données sur les soins ambulatoires, et supérieure de 20 points de pourcentage aux algorithmes utilisant seulement les données sur l'hospitalisation.

L'incidence de la source des données sur la performance des algorithmes est particulièrement évidente dans les résultats donnés par les algorithmes 1, 2 et 3 . L'algorithme 3 , qui ne requiert qu'un dossier de l'une ou l'autre des deux sources pour établir un diagnostic d'ICC, affiche la sensibilité la plus élevée $(89,9 \%)$, mais la spécificité la plus faible $(93,5 \%)$ et la VPP la plus basse $(38,0 \%)$. L'utilisation des seules données sur l'hospitalisation (algorithme 1) est la méthode la moins sensible, ne permettant de repérer que 60,6 \% des cas d'ICC, mais présente également la plus grande spécificité $(98,6 \%)$ et la VPP la plus élevée $(65,9 \%$; tableau 2$)$. La valeur prédictive négative (données non présentées) est dans tous les cas élevée, allant de 99,6\% pour l'algorithme 3 à 98,2 \% pour l'algorithme 1 .

En ce qui concerne les résultats des mesures sommaires, il est intéressant de noter que les algorithmes les plus utiles sont là aussi ceux qui utilisent à la fois les données sur l'hospitalisation et celles sur les soins ambulatoires. Les algorithmes qui n'utilisent qu'un de ces types de données, qu'il s'agisse de celles sur l'hospitalisation ou de celles sur les soins

\section{TABLEAU 2}

Sensibilité, spécificité et VPP de neuf algorithmes utilisés pour estimer le statut à l'égard de l'ICC à partir de données administratives et de données de médecins de premier recours extraites manuellement

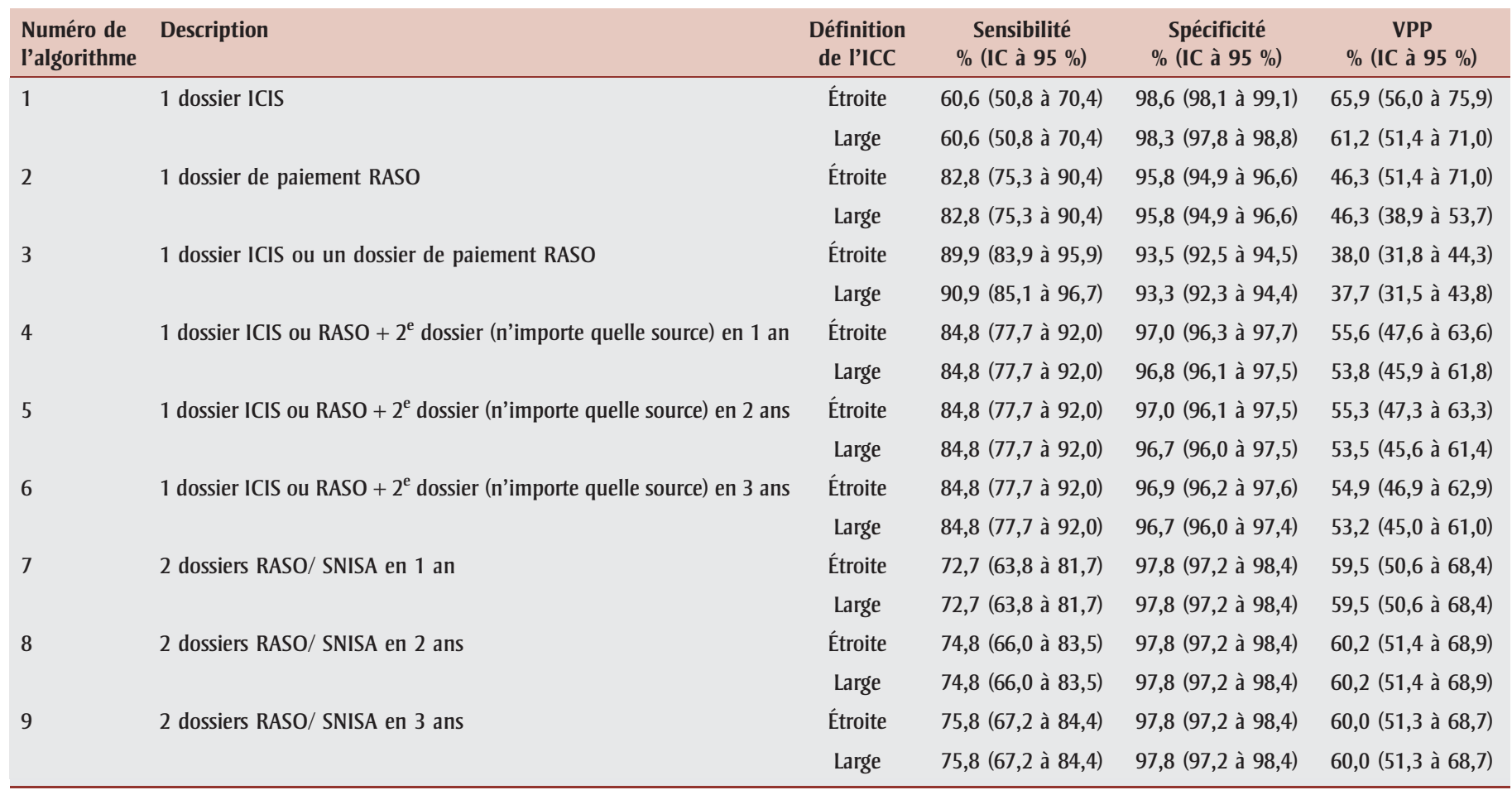

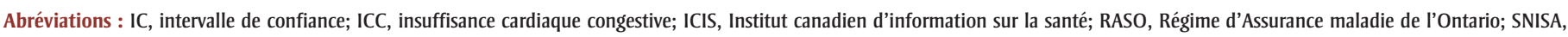
Système national d'information sur les soins ambulatoires; VPP, valeur prédictive positive. 
FIGURE 1

Représentation sous forme de courbe ROC des résultats de neuf algorithmes visant à repérer les cas d'insuffisance cardiaque congestive (ICC)

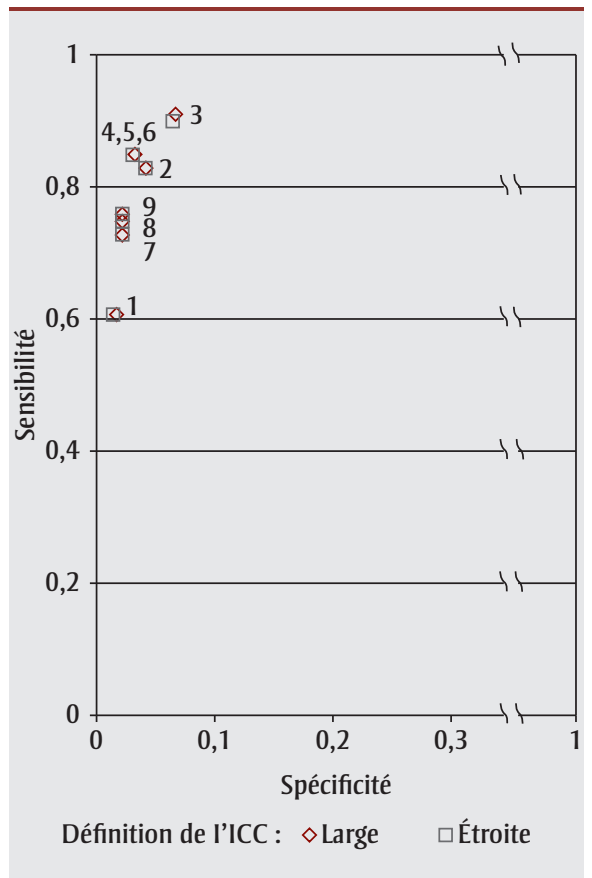

Abréviations : ICC, insuffisance cardiaque congestive; ROC, receiver operating characteristic (caractéristique de la performance d'un test). ambulatoires, sont tous situés à un point inférieur de la courbe ROC ou dans la région «faible utilité » du graphique du $\mathrm{RV}$ (figure 1). En ce qui concerne la courbe ROC (figure 2), l'algorithme 3 puis les algorithmes 4,5 et 6 présentent les meilleures performances parmi les neuf. L'ASC est égale à 0,917 pour l'algorithme 3 et à 0,909 pour les algorithmes 4,5 et 6 . Si l'on compare les résultats sur le plan du RV (figure 2), les algorithmes 3, 4, 5, 6 et 2 se situent tous dans la section « utilité moyenne » du graphique, l'algorithme 3 étant le plus proche de la section « grande utilité », et l'algorithme 2, le plus éloigné de celle-ci.

\section{Choix du meilleur algorithme}

Le choix final du meilleur algorithme pour repérer les cas d'ICC devait se faire entre les algorithmes 3 et 4 (les algorithmes 5 et 6 ayant été exclus en raison de l'inutilité de la longue période de suivi qui leur est associée). D'après les résultats des mesures sommaires, l'algorithme 3 semblait être supérieur. De surcroît, si ce test devait être utilisé dans un cadre clinique, il serait en général le plus performant, principalement parce que sa sensibilité élevée réduit au minimum le nombre de cas susceptibles d'être manqués. Toutefois, pour les études représentatives de la population, la proportion de faux positifs est un critère beaucoup plus important. Comme cet algorithme doit être utilisé pour l'ensemble d'une population, et pas simplement pour certains patients qui choisissent volontairement de consulter un médecin, et comme il s'agit d'une affection relativement rare, une légère diminution de la spécificité peut suffire pour produire un grand nombre de faux positifs. Les faux positifs, à leur tour, mènent à des résultats de recherche biaisés en faveur de l'hypothèse nulle. Par conséquent, l'algorithme 3 a été écarté en raison de sa spécificité et de son $\mathrm{RV}+$ relativement faibles. Le meilleur algorithme pour repérer les cas d'ICC est finalement l'algorithme 4.

En plus d'évaluer les différents algorithmes, nous avons également mis à l'épreuve deux définitions différentes de l'ICC. Dans la plupart des cas, il n'y a eu que peu de différences dans les résultats. Dans les cas où une différence a été observée, la définition étroite avait une meilleure performance, principalement

FIGURE 2

Rapports de vraisemblance (RV) pour neuf algorithmes visant à repérer les cas d'insuffisance cardiaque congestive (ICC)

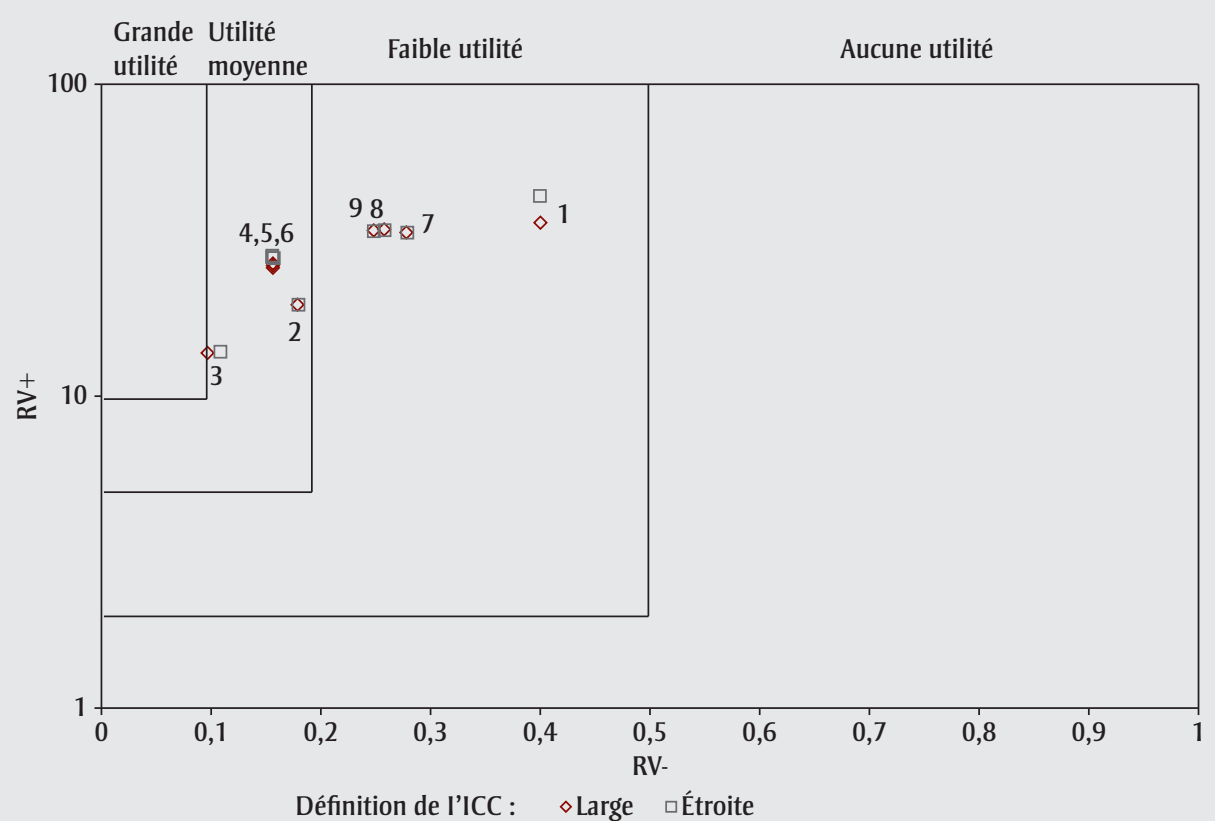

Abréviation : RV, rapport de vraisemblance. 
parce que l'utilisation de la définition plus large tendait à engendrer un plus grand nombre de faux positifs (tableau 3).

\section{Analyse}

Dans cette étude, nous avons mis à l'épreuve neuf algorithmes de données administratives variant du point de vue du nombre de dossiers requis pour un diagnostic d'ICC, de la durée de la période de suivi autorisée et des sources des données utilisées. Nous avons également mis à l'épreuve deux ensembles différents de codes de diagnostic utilisés pour repérer les individus atteints d'ICC dans les données administratives.

Dans les études précédentes portant sur la comparaison de l'information figurant dans les dossiers des hôpitaux et des données sur les congés, on avait conclu que le codage des diagnostics d'ICC donnait des résultats d'une grande exactitude par rapport à des critères cliniques comme le score de Framingham ou les critères de Boston ${ }^{4,5}$. Toutefois, nous avons constaté que les données sur les hospitalisations seules étaient insuffisantes pour estimer la prévalence de
l'ICC et risquaient d'entraîner l'omission de $40 \%$ des cas positifs. Cela laisse à penser qu'une proportion appréciable d'Ontariens atteints d'ICC reçoivent leur diagnostic et sont pris en charge à l'extérieur de l'hôpital.

Une autre observation générale est que le fait de fonder un diagnostic d'ICC sur un seul dossier contenant un code de diagnostic d'ICC peut permettre de repérer les individus atteints de cette affection dans la mesure où l'on utilise à la fois les données sur l'hospitalisation et celles sur les soins ambulatoires. Toutefois, cette méthode donne lieu à un nombre de faux positifs beaucoup trop élevé pour être acceptable. Il est possible qu'un médecin inscrive le code de l'ICC alors qu'il exclut cette maladie, auquel cas un second code de diagnostic serait nécessaire pour confirmer qu'il s'agit bien d'un cas d'ICC.

Nous avons trouvé que le meilleur algorithme pour repérer les cas d'ICC est l'utilisation d'un dossier d'hospitalisation seul, ou d'un dossier de soins ambulatoires s'il est suivi d'un second dossier de l'une ou l'autre source en l'espace d'un an; cette méthode permet de repérer environ $85 \%$ des patients atteints d'ICC, tout en maintenant le taux de faux positifs à environ $3 \%$, ce qui est relativement peu élevé. Cette observation est similaire à celle faite dans des études portant sur d'autres maladies chroniques comme le diabète $^{7}$, l'hypertension ${ }^{6}$ et la cardiopathie ischémique ${ }^{8}$, ainsi que dans des études de validation antérieures portant sur l'utilisation de données administratives pour repérer les cas d'ICC ${ }^{10,11}$

L'absence quasi totale d'effet de la durée de la période de suivi jusqu'au second dossier d'ICC a un peu surpris, car elle n'avait pas été observée dans des études similaires portant sur l'hypertension et le diabète $^{6,7}$. Ce phénomène peut s'expliquer par le fait que la plupart des personnes réellement atteintes d'ICC prennent des médicaments, ce qui les amène selon toute vraisemblance à consulter un médecin à des intervalles de quelques mois. Par conséquent, un suivi d'un an est suffisant pour que notre algorithme permette de repérer les cas d'ICC, même si les patients ne sont pas hospitalisés. En revanche, l'hypertension et le diabète peuvent au départ être pris en charge sans médicament, ce qui peut amener les personnes

TABLEAU 3

Comparaison du statut à l'égard de l'insuffisance cardiaque obtenu à partir de données administratives et de données de médecins de premier recours extraites manuellement à l'aide de neuf algorithmes (nombre)

\begin{tabular}{|c|c|c|c|c|c|c|}
\hline Algorithme & Description & Définition de I'ICC & Vrais positifs $\mathrm{N}$ & Faux positifs $\mathrm{N}$ & Vrais négatifs $\mathbf{N}$ & Faux négatifs $\mathrm{N}$ \\
\hline \multirow[t]{2}{*}{1} & \multirow[t]{2}{*}{1 dossier ICIS } & Étroite & 60 & 31 & 2208 & 39 \\
\hline & & Large & 60 & 38 & 2201 & 39 \\
\hline 2 & 1 dossier de paiement RASO & Large & 82 & 95 & 2144 & 17 \\
\hline 3 & 1 dossier ICIS ou RASO & Étroite & 89 & 145 & 2094 & 10 \\
\hline 5 & $\begin{array}{l}1 \text { dossier ICIS ou RASO }+2^{\mathrm{e}} \text { dossier } \\
\text { (n'importe quelle source) en } 2 \text { ans }\end{array}$ & $\begin{array}{l}\text { Étroite } \\
\text { Large }\end{array}$ & $\begin{array}{l}84 \\
84\end{array}$ & $\begin{array}{l}68 \\
73\end{array}$ & $\begin{array}{l}2171 \\
2166\end{array}$ & $\begin{array}{l}15 \\
15\end{array}$ \\
\hline 6 & $\begin{array}{l}1 \text { dossier ICIS ou RASO }+2^{\mathrm{e}} \text { dossier } \\
\text { (n'importe quelle source) en } 3 \text { ans }\end{array}$ & $\begin{array}{l}\text { Étroite } \\
\text { Large }\end{array}$ & $\begin{array}{l}84 \\
84\end{array}$ & $\begin{array}{l}69 \\
74\end{array}$ & $\begin{array}{l}2170 \\
2165\end{array}$ & $\begin{array}{l}15 \\
15\end{array}$ \\
\hline 8 & 2 dossiers RASO/ SNISA en 2 ans & Large & 74 & 49 & 2190 & 25 \\
\hline \multirow[t]{2}{*}{9} & \multirow[t]{2}{*}{2 dossiers RASO/ SNISA en 3 ans } & Étroite & 75 & 50 & 2189 & 24 \\
\hline & & Large & 75 & 50 & 2189 & 24 \\
\hline
\end{tabular}

Abréviations : ICC, insuffisance cardiaque congestive; ICIS, Institut canadien d'information sur la santé; RASO, Régime d'Assurance maladie de I'Ontario; SNISA, Système national d'information sur les soins ambulatoires. 
atteintes à consulter moins fréquemment leur médecin; dans de telles circonstances, une période de suivi plus longue est requise avant l'obtention d'un second dossier.

Pour tous les algorithmes, la VPP semble plutôt faible, variant de 37,7 à 65,9. Cela tient au fait que la VPP est étroitement liée à la prévalence : plus la prévalence est faible, plus la VPP est faible. L'ICC étant relativement rare dans notre population, avec une prévalence de 4,3\% seulement, les valeurs de la VPP sont elles aussi peu élevées.

L'ICC est étroitement liée à l'âge : alors qu'elle est extrêmement rare chez les moins de 40 ans et rare chez les individus de 40 à 65 ans, sa prévalence augmente graduellement après 65 ans. Cela nous incite à recommander que l'algorithme ne soit pas utilisé pour les populations où l'on sait que l'ICC est très rare (c'est-à-dire chez les moins de 40 ans). On peut s'attendre à ce que l'algorithme donne de très bons résultats chez les personnes âgées, en particulier chez celles de plus de 75 ans.

En ce qui concerne les codes de la CIM-9 et de la CIM-10 utilisés pour définir l'ICC, nous n'avons trouvé aucune preuve indiquant que l'utilisation d'une liste élargie de codes de diagnostic donnait de meilleurs résultats que la définition étroite de la maladie par le code 428 de la CIM- 9 et les codes I500, I501 et I509 de la CIM-10.

\section{Limites}

Les limites de cette étude tiennent surtout au fait que la plupart des données utilisées ont été recueillies à des fins administratives et non à des fins de recherche. Une limite importante des données de l'assurance maladie de l'Ontario est qu'un seul code de diagnostic figure sur chaque facture. Si l'ICC n'est pas la raison principale de la consultation du médecin, elle peut ne pas être consignée. Cette limite est compensée par le fait que les Ontariens consultent assez fréquemment leur médecin, en particulier s'ils ont plus de 65 ans, ce qui offre au médecin plusieurs occasions de noter l'ICC sur une facture en l'espace d'une année, comme le montre la constatation que la durée du suivi n'a aucun effet sur la capacité de nos algorithmes de repérer les cas d'ICC.

Si nous reconnaissons que nous n'avons pas pu appliquer les critères diagnostiques de la New York Heart Association (NYHA) à l'ICC pour déterminer la présence d'une insuffisance cardiaque, nous avons cependant utilisé des données du «monde réel » produites par les actes de diagnostic et de prise en charge des médecins en fonction de leur propre acuité diagnostique. Cet aspect ne s'évalue pas facilement selon des critères structurés et formels, mais nous sommes d'avis que si un médecin traite un patient pour une ICC, il est raisonnable de considérer que le patient est atteint de cette affection pour ce qui concerne le calcul de son incidence et de sa prévalence dans une population importante.

\section{Conclusion}

Dans cette étude, nous avons montré qu'il est possible de repérer les cas d'ICC avec un degré d'exactitude élevé à partir de données administratives, dans la mesure où l'on utilise à la fois les dossiers sur les hospitalisations et ceux sur les soins ambulatoires. Un algorithme s'appuyant sur un dossier d'hospitalisation ou sur un dossier de soins ambulatoires suivi d'un second dossier de l'une ou l'autre source dans l'année, l'ICC étant définie par le code de diagnostic 428 de la CIM-9 et les codes I500, I501 et I509 de la CIM-10, permet de repérer les patients atteints d'ICC avec une sensibilité de $84,8 \%$ et une spécificité de 97,0\%.

\section{Remerciements}

Cette étude a bénéficié de l'appui de l'Institut de recherche en services de santé (IRSS), qui est financé par une subvention annuelle du ministère de la Santé et des Soins de longue durée de l'Ontario. Les opinions, résultats et conclusions de l'étude n'engagent que ses auteurs et sont indépendants des sources de financement. Ils ne sont nullement le reflet de la position de l'IRSS ou du ministère de la Santé et des Soins de longue durée de l'Ontario.

La $D^{\text {re }}$ Karen $\mathrm{Tu}$ est récipiendaire d'une bourse de recherche en soins de santé primaires des Instituts de recherche en santé du Canada (IRSC).

Cette étude a bénéficié d'une subvention de l'Agence de la santé publique du Canada et d'une subvention d'équipe pour la recherche sur les maladies vasculaires attribuée par les IRSC à l'Équipe canadienne de recherche sur les résultats des interventions en santé cardiovasculaire.

Conflit d'intérêts : aucun.

\section{Références}

1. Lee DS, Johansen H, Gong $\mathrm{Y}$ et collab. Regional outcomes of heart failure in Canada. Can J Cardiol. 2004;20(6):599-607.

2. Hall RE, Tu JV. Hospitalization rates and length of stay for cardiovascular conditions in Canada, 1994 to 1999. Can J Cardiol. 2003;19(10):1123-31.

3. Saczynski JS, Andrade SE, Harrold LR et collab. A systematic review of validated methods for identifying heart failure using administrative data. Pharmacoepidemiol Drug Saf. 2012;21(S1):129-40.

4. Lee DS, Donovan L, Austin PC et collab. Comparison of coding of heart failure and comorbidities in administrative and clinical data for use in outcomes research. Med Care. 2005;43(2):182-8.

5. Teng TH, Finn J, Hung $J$ et collab. A validation study: how effective is the Hospital Morbidity Data as a surveillance tool for heart failure in Western Australia? Aust N Z J Public Health. 2008;32(5):405-7.

6. Tu K, Campbell NR, Chen ZL et collab. Accuracy of administrative databases in identifying patients with hypertension. Open Medicine. 2007;1(1):E18-26.

7. Hux JE, Ivis F, Flintoft $V$ et collab. Diabetes in Ontario: determination of prevalence and incidence using a validated administrative data algorithm. Diabetes Care. 2002;25(3): 512-6.

8. Tu K, Mitiku T, Lee DS et collab. Validation of physician billing and hospitalization data to identify patients with ischemic heart disease using data from the Electronic Medical Record Administrative data Linked Database (EMRALD). Can J Cardiol. 2010;26(7):e225-8. 
9. Gershon AS, Wang C, Guan J et collab. Identifying individuals with physician diagnosed COPD in health administrative databases. COPD. 2009;6(5):388-94.

10. Alqaisi F, Williams LK, Peterson EL et collab. Comparing methods for identifying patients with heart failure using electronic data sources. BMC Health Serv Res. 2009;9:237.

11. McCullough PA, Philbin EF, Spertus JA et collab. Confirmation of a heart failure epidemic: findings from the Resource Utilization Among Congestive Heart Failure (REACH) study. J Am Coll Cardiol. 2002;39(1):60-9.

12. Chan BT, Schultz SE. Supply and utilization of general practitioner and family physician services in Ontario. ICES Investigative Report. Toronto (Ont.): Institute for Clinical Evaluative Sciences; 2005.

13. Vermeulen MJ, Tu JV, Schull MJ. ICD-10 adaptations of the Ontario acute myocardial infarction mortality prediction rules performed as well as the original versions. J Clin Epidemiol. 2007;60(9):971-4.

14. Li B, Evans D, Faris P et collab. Risk adjustment performance of Charlson and Elixhauser comorbidities in ICD-9 and ICD10 administrative databases. BMC Health Serv Res. 2008;8:12.

15. Chen G, Faris P, Hemmelgarn Bet collab. Measuring agreement of administrative data with chart data using prevalence unadjusted and adjusted kappa. BMC Med Res Methodol. 2009;9:5.

16. Ontario Health Technology Advisory Committee. Multi-detector computed tomography angiography for coronary artery disease. Toronto (Ont.): Ministry of Health and Long-Term Care; 2005.

17. Jaeschke R, Guyatt GH, Sackett DL. Users' guides to the medical literature. III. How to use an article about a diagnostic test. B. What are the results and will they help me in caring for my patients? The EvidenceBased Medicine Working Group. JAMA. 1994;271(9):703-7.

18. Canada. Statistique Canada. Estimations démographiques annuelles : Canada, provinces et territoires, 2009. Ottawa : Statistique Canada; 2009 [Statistique Canada, n॰ 91-215-X au catalogue].
19. Bertakis KD, Azari R, Helms LJ et collab. Gender differences in the utilization of health care services. J Fam Pract. 2000; 49(2):147-52. 